$$
\text { CONF-951155--111 }
$$

Note: This is a preprint of a paper submitted for publication. Contents of this paper should not be quoted or referred to without permission of the author(s).

Presented at Fall Meeting of the Materials Research Society, Boston, Massachusetts, November 27-December 1, 1995 and to be published in Evolution of Epitaxial Structure and Morphology, ed. by A. Zangwill, D. Jesson, D. Chambliss, and R. Clarke, Materials Research Society, Pittsburgh, Pennsylvania

\title{
SELF-LIMITING GROWTH KINETICS OF 3D COHERENT ISLANDS
}

K. M. Chen, D. E. Jesson, S. J. Pennycook, T. Thundat,* and R. J Warmack*

Solid State Division, Oak Ridge National Laboratory

Oak Ridge, Tennessee 37831-6030

*Health Sciences Research Division, Oak Ridge National Laboratory

Oak Ridge, Tennessee 37831-6123

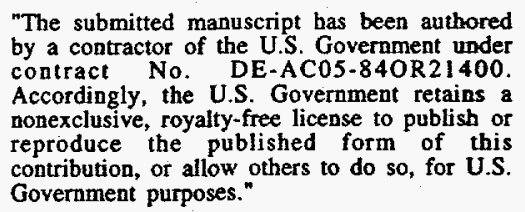

"The submitted manuscript has been authored

by a contractor of the U.S. Government under

contract No. DE-AC05-840R21400.

Acrexclusive, royalty-free license to publish or

reproduce the published form of this

Government purposes."

\section{Prepared by}

SOLID STATE DIVISION

OAK RIDGE NATIONAL LABORATORY

Managed by

LOCKHEED MARTIN ENERGY SYSTEMS

under

Contract No. DE-AC05-84OR21400

with the

U.S. DEPARTMENT OF ENERGY

Oak Ridge, Tennessee

December 1995 


\author{
K. M. CHEN*, D. E. JESSON*, S. J. PENNYCOOK*, T. THUNDAT**, AND R. J. \\ WARMACK** \\ * Solid State Division, Oak Ridge National Laboratory, Oak Ridge, TN 37831-6031; \\ ** Health Sciences Research Division, Oak Ridge National Laboratory, Oak Ridge, TN 37831
}

\title{
ABSTRACT
}

Large 3D coherent islands are found to kinetically resist their further growth during postdeposition annealing of metastable 2D strained films. We reveal that a kinetic energy barrier exists to successive facet-layer growth of a 3D strained island at the expense of its surrounding 2D structure. The barrier increases with further growth of the island, which defines a self-limiting behavior of island growth. This self-limiting effect naturally explains a number of relevant features which have been observed experimentally including narrow island size distributions.

\section{INTRODUCTION}

Quantum-confined semiconductor structures constitute very attractive objects both for their fundamental properties and their potential applications in micro- and opto-electronics. ${ }^{1}$ While quantum well structures are already widely used in many devices, quantum wires and quantum dots are much more difficult to fabricate for this purpose. Three dimensional (3D) coherent islands, which are formed during deposition of a highly mismatched film on a planar low-index substrate, have recently been demonstrated to exhibit promising quantum confinement effects from individual islands. 1 Therefore, the growth of 3D islands may provide an in-situ approach to the fabrication of quantum dots. However, the success of this approach critically relies on our ability to control the size, shape and uniformity of the 3D islands, as their optical and electronic properties sensitively depend on these parameters. Hence it is crucially important to understand the general physical principles governing the growth kinetics of 3D strained islands.

It is well known that, 3D island formation is intrinsically driven by the misfit stress between the epitaxial film and substrate as it can elastically relieve the stress via deformation of the substrate. ${ }^{2}$ Thermodynamically, 3D islands are self-assembled and self-organized. Here we reveal another important and fascinating feature, the self-limiting behavior of 3D strained island growth, which is highly relevant to the fabrication of monosized quantum dots.

Over the past three years, several groups ${ }^{3-5}$ have reported a remarkably narrow size distribution of 3D islands, which were observed after molecular beam epitaxy (MBE) deposition of a few monolayer coverage of a highly strained InAs film on a GaAs substrate (7\% mismatch). However, the mechanism responsible for this narrow size distribution has not been well understood. A few energetic models 6-8 have been proposed to explain this feature. In all of these models, a global thermodynamic equilibrium state was assumed for the growth of either twodimensional (2D) strained islands, or 3D coherent islands, somewhat contrary to the nonequilibrium nature of MBE growth processes. To reveal the mechanism responsible for the sizeuniformity, we investigate 3D island growth from a metastable 2D strained film, which is induced by a post-deposition annealing. This approach emulates equilibrium surface conditions, at least locally, as close as possible. Our results determine that the high size-uniformity can result from a self-limiting behavior during the growth stage, i.e. well before a global equilibrium state is reached.

\section{EXPERIMENTAL OBSERVATIONS}

We began with a flat $2 \mathrm{~nm}$ thick $\mathrm{Ge}_{0.5} \mathrm{Si}_{0.5}$ strained film (2\% mismatch) which was deposited on Si(100) substrate at low temperature of $400^{\circ} \mathrm{C}$. The flat film surface was unstable to the formation of 3D islands upon post-deposition annealing for temperatures above a transition temperature region around $570^{\circ} \mathrm{C}$. Since the chemical potential of a stable 3D island is lower than that of a $2 \mathrm{D}$ film, the planar strained film can be treated as an atom reservoir for 3D island 
nucleation and growth. The islands at this growth stage were examined by atomic force microscopy (AFM) after quenching samples from different annealing stages. Taking advantage of the existence of a temperature gradient across the sample surface $\left(20 \pm 5^{\circ} \mathrm{C}\right.$ difference from the sample center to edge), we can obtain information about the growth evolution from AFM measurements of islands at different temperature regions of one sample wafer. Fig.la and $b$ show the typical AFM images of islands at the edge and center regions, respectively. Most of the islands have strain-stabilized (501) facet planes. The number density of strained 3D islands in (b) is much higher than that in (a), consistent with the thermally activated nature of $3 \mathrm{D}$ island formation. 8.9

A surprising feature revealed in fig. $1 \mathrm{~b}$ is the uniformity of island sizes, which can be clearly seen from the remarkably narrow distribution shown in fig.2. Comparing the size distributions between (a) and (b), we find that the size distribution is rather broad at initial stage of $3 D$ island formation, but narrows upon further annealing. More surprisingly, however, is that the maximum sizes in both distribution curves are almost unchanged. From the count of total volume of 3D islands, we know that the 2D atom reservoir still exists surrounding each 3D island. These results indicate that the narrow size distribution results from the resistance of large individual islands to further growth (i. e. slower growth rate), while small sized islands continue to grow, eventually catching up in size with the large ones. This scenario is also consistent with the change in the curve shapes of the size distributions, in which the right shoulder of the distribution curve becomes much narrower upon island growth. A similar feature was also observed during a low supersaturation growth of InAs strained islands on GaAs, 3,4 in which the increase of InAs coverage was found to result in the increase of island density rather than the size of each island. Therefore, we believe that the self-resistance feature may be general to all strained systems.
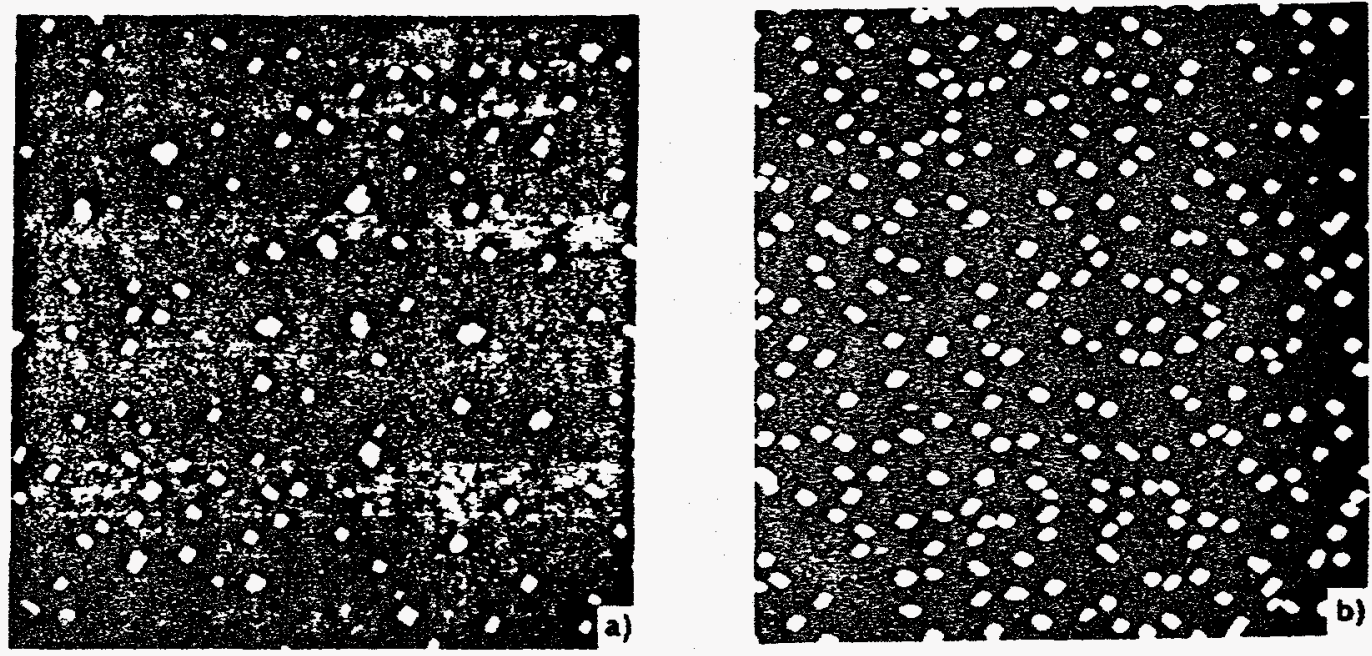

Fig. 1 AFM images of 3D islands at (a) edge region, and (b) center area of a quenched sample wafer, which has been annealed at (center) temperature of $590^{\circ} \mathrm{C}$ for 6 minutes after the deposition of a flat, $2 \mathrm{~nm}$ thick $\mathrm{Ge}_{0.5} \mathrm{Si}_{0.5}$ strained film on a Si(001) substrate. The temperature at the edge was about $20^{\circ} \mathrm{C}$ lower that at center. The scan size of the images is $2 \times 2 \mu \mathrm{m}^{2}$, and the black to white contrast corresponds to a height scale ranging from 0 to $10 \mathrm{~nm}$.

When the sample was annealed at a much higher temperature $\left(\geq 650^{\circ} \mathrm{C}\right)$ for 10 minutes, and the atom reservoir was exhausted, a much broader distribution in island size was observed, as shown in Fig. 3. Larger islands with half width sizes of $60 \mathrm{~nm}$ were also achieved, and several coalescence events could be identified from AFM measurements. This clearly indicates the islands were undergoing a coarsening process, in which larger islands grew at the expense of small ones via Ostwald ripening and/or coalescence events. This fact suggests that the resistance of island growth during the growth stage is a kinetic effect, rather than due to the thermodynamics of the 3D island system. 


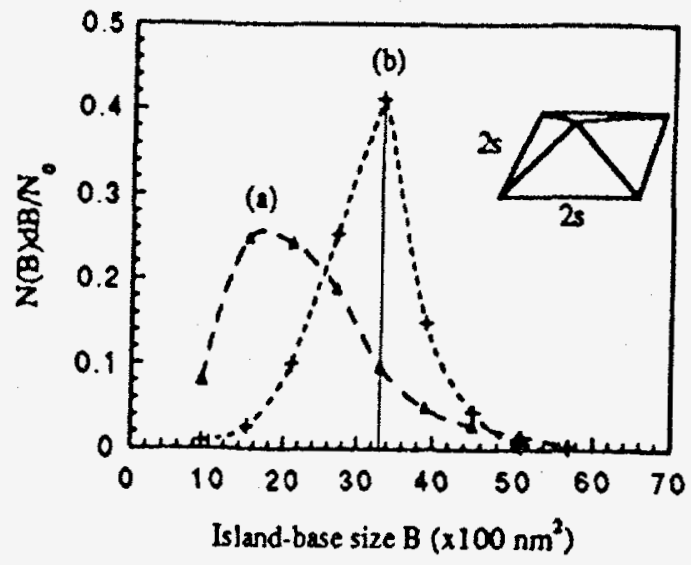

Fig. 2 Size distributions of the samples in figure $l$ as a function of island-base size $B$ $\left(=4 s^{2}\right)$. $N(B) d B$ represents the number of islands with sizes between $B$ and $B+d B$, and $N_{0}$ is the total number of islands surveyed.
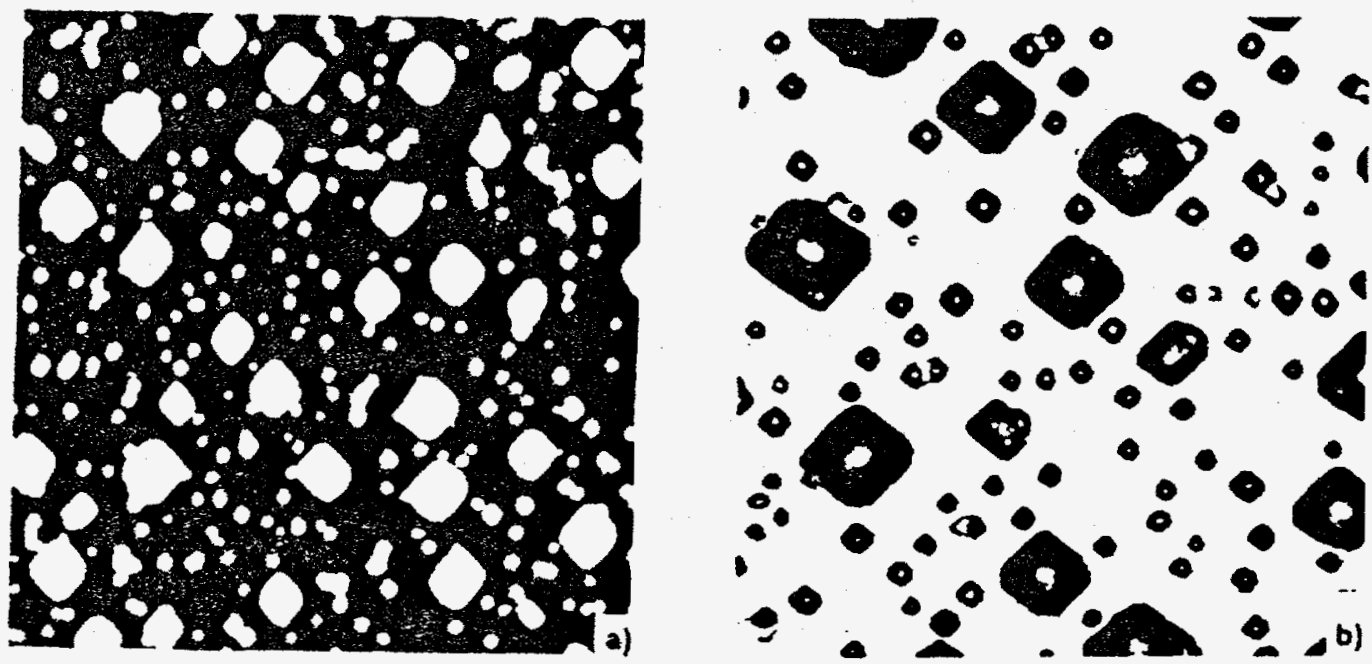

Fig. 3 AFM images of strained Ge0.5 $\mathrm{Si}_{0.5}$ islands induced by annealing for 10 minutes at a temperature of $650^{\circ} \mathrm{C}$. The scan sizes are (a) $2 \times 2 \mu \mathrm{m}^{2}$; and (b) $1 \times 1 \mu \mathrm{m}^{2}$.

\section{THEORETICAL ANALYSIS}

Now, we would like to address the basic question: Why do larger strained islands seem to kinetically resist further growth? It is very likely that the strain must play a dominant role in the growth of the islands. This leads us to carefully examine the effect of the misfit strain on both the energetics and kinetics of the 3D island growth.

The growth rate of a strained 3D island is determined by the net incoming fluxes via surface diffusion $J_{S}$ and/or via the vapor condensation $J_{V}$ (as illustrated in Fig. 4), and can be written as

$$
\frac{d n(s, \theta)}{d t}=J_{s}+J_{v}
$$

where $n(s, \theta)$ is the number of atoms in a pyramidal island with an inclination angle $\theta$ and size $s$ (see fig.6). However, $J_{v}=0$ in the case of annealing experiments, or $J_{v} \ll J_{s}$ at the initial stage of island growth when the island size is much smaller than the diffusion length. Thus the growth of an island is dominated by the flux via surface diffusion from the surrounding surface area. The mass transportation of atoms from the planar surface to the island surface involves two distinct steps: step A, surface diffusion of adatoms from a distance to near island edge, characterized by the surface diffusion coefficient $D_{s}$ of adatoms; and step $B$, the eventual transfer from the planar 
surface to the beveled island surface, characterized by a probability constant $\beta$ for adatoms moving from the planar surface to the island surface. It is a serial process and the growth rate or $J_{s}$ is determined by the slowest step. At steady state, the growth rate is 10

$$
\frac{d n(s, \theta)}{d t}=\frac{\frac{2 \pi D_{s}}{\log I} \beta A}{\frac{2 \pi D_{s}}{\log l}+\beta A}\left[C_{f}-C_{r}\right]
$$

Here the island surface area $A=4 s^{2} / \cos (\theta) ; c$ is the free adatom concentration or the equilibrium adatom concentration over a planar surface of a strained film, representing the incoming flux via surface diffusion; $C_{r}$ is the equilibrium adatom concentration of the strained island, representing the desorptive flux via surface diffusion under a hypothetic equilibrium. $C_{r}$ is given by GibbsThomson relationship, $C_{r}=C_{f} \exp (\Delta \mu / \mathrm{kT})$, where $\Delta \mu$ is the chemical potential difference between the 3D island and the 2D phase; $l^{*} s$ is a "screening" distance at which the adatom concentration recovers to $C_{f}$ from the local concentration of adatoms near island edge. In the equation, term $\left[C_{f}-C_{r}\right]$ represents the thermodynamic driving force for $3 \mathrm{D}$ island growth (if $C_{f}>C_{r}$ ) or dissolution (if $C_{f}<C_{r}$ ), while its prefactor term contains the kinetic information about the surface mass transportation for island growth or dissolving.

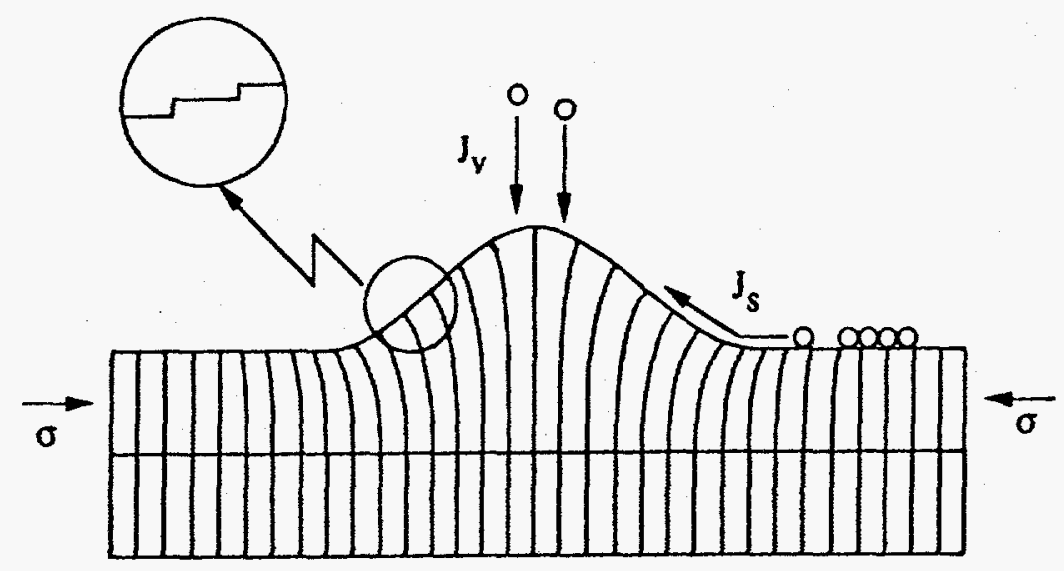

Fig. 4. A diagram showing 3D island growth via surface diffusion and/or vapor condensation.

\section{Energetics of 3D Island Growth}

We first examine the strain effect on the thermodynamic driving force, i.e. the energetics of island growth. The free energy change accompanying the formation of a 3D island from a planar film is given by $\Delta G=\Delta G_{s}-\Delta G_{r}$. The elastic relaxation energy $\Delta G_{r}$ drives the transition but is opposed by the creation of surface energy $\Delta G_{s}$. Microscopically, the facet surface of an island face constitutes regularly spaced atomic steps as shown in fig.4. Since the island facets preferentially align along the elastically soft [100] direction, the steps are primarily associated with $[100]$ type monatomic steps. ${ }^{9}$ At each step, the discontinuity in the height of the surface creates a force monopole in the projected two-dimensional stress. The elastic relaxation energy can be obtained from the interaction of these monopoles. ${ }^{8}$ On the other hand, the surface energy cost is the sum of the step creation energies and step-step repulsive interaction energy. We emphasize that the decomposition of the energies into atomic steps is validated by our observation of stable islands associated with small slopes. Approximately, we treat each step as having a length $s$, so that the total free energy change associated with a square-based pyramidal island is 8,9 


$$
\Delta G=2 s\left[\sum_{i} C_{0}+\sum_{i>j} C_{d}\left(\frac{x_{i}-x_{j}}{a}\right)^{-2}+\sum_{i>j} C_{m} s_{i} s_{j} \ln \left(\frac{\left|x_{i}-x_{j}\right|}{a}\right)\right]
$$

Here $s_{i}$ is +1 for up steps and -1 for down steps; $a$ is a microscopic cut-off length, usually taken as surface lattice constant $C_{m}=(1-v) \sigma^{2} h^{2} / \pi \mu$, where $\sigma$ is the misfit stress; $h$ is the step height; $\mu$ and $v$ are the shear modulus and Poison's ratio respectively. For a pyramidal island with perfect facet faces, we can deduce from Eq.(3) that 8,9 the elastic relaxation energy $\Delta G$ is proportional to island volume $\mathrm{V}$, while the surface energy cost $\Delta G_{s}$ is proportional to $\mathrm{V} 2 / 3$. A typical energy curve $\Delta G$ vs. $V$ is shown fig. 5 . The free energy per atomic volume, or the chemical potential of a $3 \mathrm{D}$ pyramidal island relative to that of a $2 \mathrm{D}$ stucture can be written as

$$
\Delta \mu(s)=\frac{4 \Omega}{s h}\left[C_{0}+\frac{2}{3} C_{d}\left(\frac{a \tan \theta}{h}\right)^{2}\right]-\frac{3 C_{m} \Omega \cot \theta}{h} .
$$

Here $\Omega$ is the atomic volume of the film material. From Eq.(4), we can see that, $\Delta \mu$ will be negative and decreases with the increase of island size $s$ when the size is larger than a critical nucleus size. This demonstrates that 3D islands beyond the critical nucleus size are stable and will continue to grow at the expense of the $2 \mathrm{D}$ structure $\left(C_{f}>C_{r}\right)$. In other words, there always exists a thermodynamic driving force for 3D islands to grow via surface diffusion of adatoms from the atom reservoir of the $2 \mathrm{D}$ film until a wetting layer is exposed. This energetic picture is consistent with the overall trend of the strained island growth as being experimentally observed, but does not predict any self-limiting behavior.

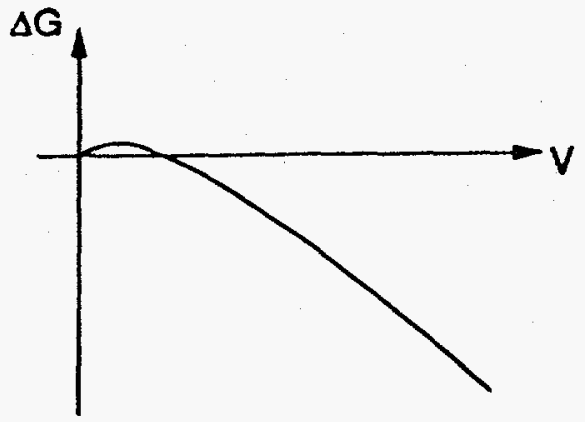

Fig. 5 A diagram showing a typical energy curve $\Delta G$ vs. volume $V$ of a pyramidal island with perfect facet surfaces.

\section{Kinetics of 3D Island Growth}

The misfit strain, however, could drastically effect the kinetics of the 3D island growth. If a coherent 3D island is well isolated and sufficiently large, there exists a large stress concentration field 2,11 along the lower part of the island surface, which will raise the local surface chemical potential to a point above that of the surrounding $2 \mathrm{D}$ atom reservoir. Therefore, for island growth via surface diffusion of atoms from the surrounding 2D structure, atoms have to experience this high surface stress region before entering the elastically relaxed region, i.e. the upper part of the island surface where the surface chemical potential is much lower. Considering the 3D island and surrounding 2D structure as an isolated system, the system energy would initially increase as the atoms of the 2D structure move to the highly stressed region. This high potential region can be treated as an energy barrier region for the growth of 3D islands, which will assume more severe self-limiting effect for the growth of faceted islands. 

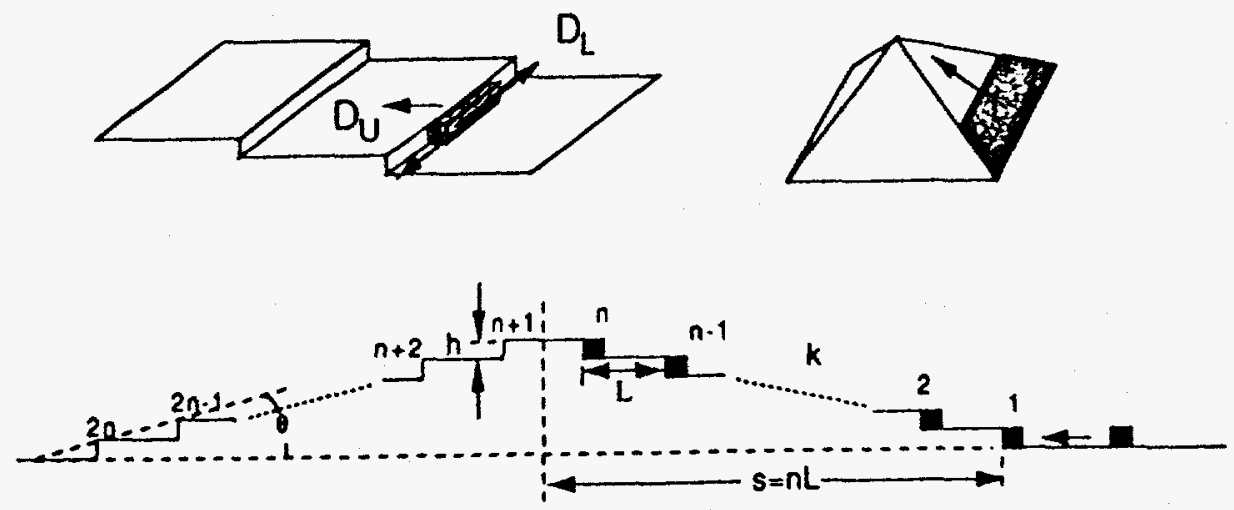

Fig. 6 Diagrams of the proposed kinetic model for 3D strained island growth. The island shape is assumed as a square-based pyramid with size $s$ and inclination angle $\theta$. A kinetic pathway is assumed to be ledge-by-ledge growth upwards from island base as discussed in the text.

We first examine the kinetic pathway for the growth of faceted islands. Although the inclination angle of GeSi islands was found ${ }^{9}$ to increase continuously at the initial stage of island growth, it would be stabilized at a facet angle around $11.2^{\circ}$ (\{501\} plane). This is because the contribution of step-step repulsive energy dominates the total island energy of Eq. (3), so that increasing the inclination angle beyond (501) facets will be energetically unfavorable, eventually leading to the formation of biatomic height steps which are associated with a high formation energy. Therefore, the (501) facets are a natural result of the (metastable) balance between the elastic relaxation energy and the energy cost associated with surface step configurations. For simplicity, we assume all islands could quickly reach this facet angle, and treat the inclination angle as a constant in the calculation.

From the above discussion, it is clear that facet islands must grow by adding (501) facets. The completion of a new face involves the nucleation and then rapid growth of a critical nucleus. There is a minimum-energy pathway for the facet layer growth as follows: a block of material from the 2D structure is first grown onto the lowest step edge labeled as 1 and complete the ledge; then second block is subsequently attached to the second lowest step edge labeled as 2; and so on up to the $n$th step. Assuming each addition of the block causes the step terrace to extend a length of $b=(\sqrt{2} / 2) a$, then one complete monolayer of block material will cover the island facet after the whole procedure, as shown in Fig. 6 . Such a sequence of growth mimics the mass transportation of atoms from the surrounding 2D structure via surface diffusion, while the island still keeps the same facet plane after successive growth of each monolayer on one of the island facet surfaces to maintain a local equilibrium. Any change in the growth sequence (for example, growing $i$ th step before growing the (i-I)th step, or growing 2 blocks at an intermediate step rather than 1 complete block) are found to cost a large step-step repulsive energy, which reflects the metastable nature of the facet surface. The kinetic picture also implies that the growth of each step ledge is completed before the next upper step start to grow. This can be justified because diffusion for atoms to "climb" upwards over steps is much slower than the lateral diffusion of atoms along the terrace edge. Estimation based on simple bond counting arguments indicates that the lateral growth rate $\mathrm{D}_{\mathrm{L}}$ of the ledge is at least two orders of magnitude larger than the vertical diffusion rate $\mathrm{D}_{\mathrm{u}}$. Therefore, both local energetics and growth kinetics imply that the facet will grow ledge-by-ledge from the base upwards.

Based on the above simple kinetic model, we calculate the change of 3D island energy during the growth of successive layers on an island facet surface. The free energy change of the strained system accompanying the addition of the ith block can be written from Eq. (3), including the extra surface energy $\operatorname{cost}\left(\gamma_{a}\right)$ at facet corners, as 


$$
\begin{aligned}
\Delta E_{k} & \left.=s C_{m}\left(\sum_{i=k}^{n-k} \ln \left(1+\frac{b}{i l}\right)-\sum_{i=1}^{n} \ln \left[1+\frac{b}{(n-k+i) l}\right]\right]-s C_{d} \sum_{i=k}^{2 n-k}\left[\left(\frac{a}{i l}\right)^{2}-\left(\frac{a}{i l+b}\right)^{2}\right]+\gamma_{a}, \quad \text { (if } k \leq n / 2\right) \\
& \text { or } \\
& s C_{m}\left(-\sum_{i=n-k}^{k} \ln \left(1+\frac{b}{i l}\right)-\sum_{i=1}^{n} \ln \left[1+\frac{b}{(n-k+i) l}\right]\right]-s C_{d} \sum_{i=k}^{2 n-k}\left[\left(\frac{a}{i l}\right)^{2}-\left(\frac{a}{i l+b}\right)^{2}\right]+\gamma_{a}, \quad(\text { if } k>n / 2)
\end{aligned}
$$

where the island size $s=n l$ and the step spacing $l=h \cot \theta$. The total change of the free energy
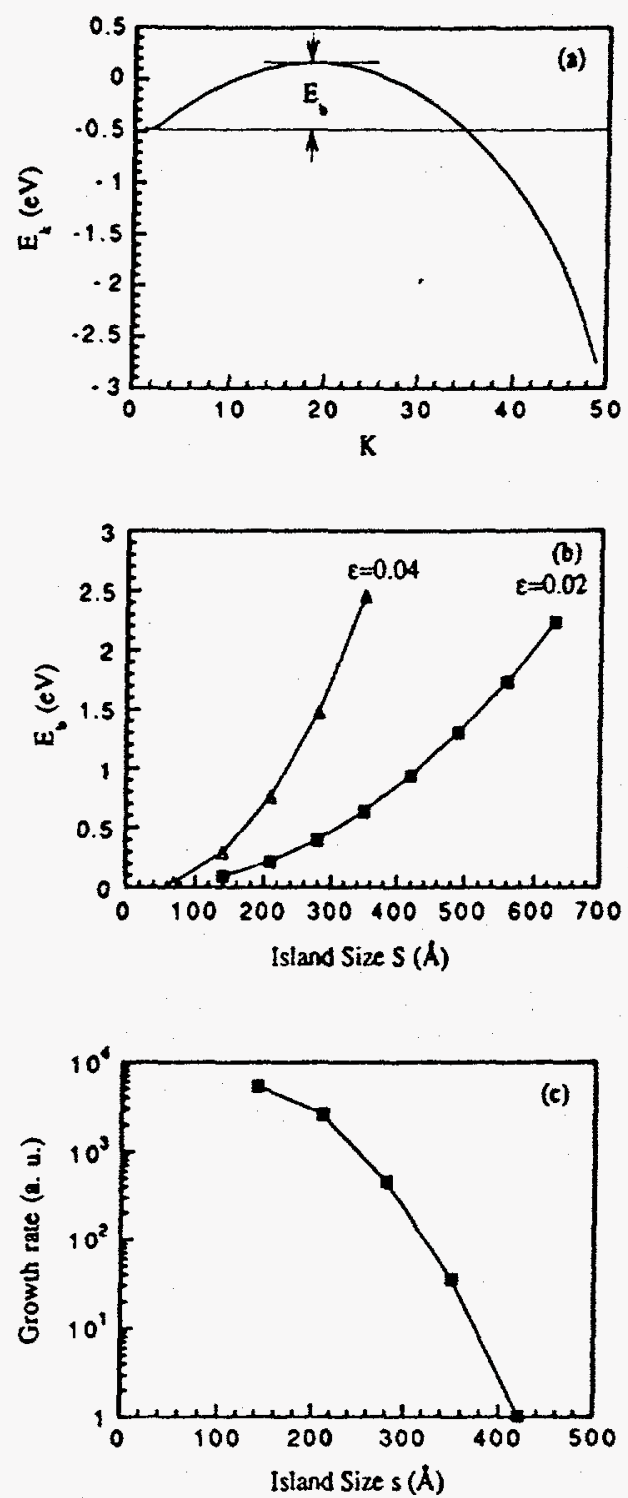

up to the $k$ th step is $E_{k}=\sum_{i=1}^{k} \Delta E_{i}$. We calculate $E_{k}$ as a function of $k$ for $k=1$ to $n$. A typical plot of the energy curve is shown in fig.7a. For sufficiently large islands, we observe an energy maximum, i.e. energy barrier $E_{b}$, at the $k^{*}$ th step of the island growth. When the island grows beyond the $k^{*}$ th step located within the elastically relaxed region, the energy drops. The system always gains energy from the elastic relaxation at the top part of the island surface after a coverage of one complete monolayer, consistent with the above energetic argument.

The existence of an energy barrier $\mathrm{E}_{b}$ indicates the thermal activated nature of successive layer growth of $3 D$ islands. This demonstrates that the growth of the 3D island involves a nucleation process. It is the cooperative nature of the nucleation process that dominates the self-limiting growth kinetics of island facets. As shown in fig. $7 \mathrm{~b}$, the energy barrier is significant only when the island is sufficiently large, and will increase with the increase of island size. This can be simply understood since bigger coherent islands are associated with a larger integrated local stress concentration at the island base, and thus have larger energy barriers. Figure 8 represents the trend of the total system energy as a function of island volume during the growth of successive facets.

Fig.7 (a) A typical plot of $E_{k}$ vs. $k$; In the calculation, we assume $\varepsilon=2 \%, C_{d}=10$ $\mathrm{meV} / \AA, h=1.4 \AA, a=3.86 \AA, \theta=11.2^{\circ}, \gamma_{a}=0.02 \mathrm{eV}$, and $n=50$; (b) The kinetic energy barrier $\mathrm{Eb}_{\mathrm{b}}$ as a function of island size $s(n)$ under different misfit strain $\varepsilon$; (c). The growth rate vs. island size. 
We estimate the growth rate of an island of size $s$ to be proportional to $s^{3} * \exp \left[-E_{b}(s) / k T\right]$. Clearly, the growth rate of an island will rapidly reduce with increase of the island size as shown in Fig. 7c, even though there exists a large thermodynamic driving force for its growth. This naturally explains the self-limiting behavior of strained island growth, i.e., the kinetic resistance of large islands to further growth. Although the calculation is rather qualitative in nature due to the approximation involved in the derivation of Eq. (3) and uncertainties in our knowledge of step parameters, it should capture the essential physical principles of the self-limiting kinetics.

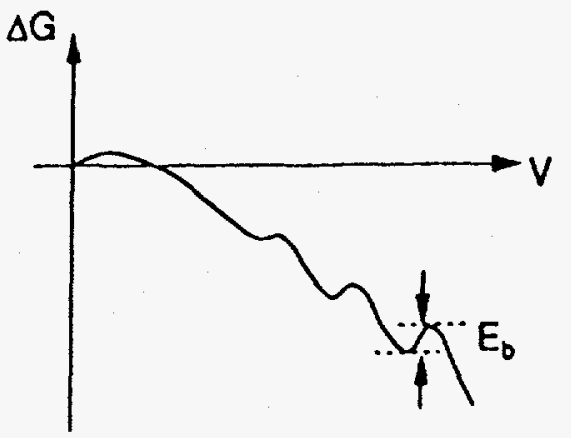

Fig. 8 A schematic diagram showing a typical energy curve during layer-by-layer growth of island facets. Note the undulations of the energy curve, which induce kinetic barriers for island growth.

\section{DISCUSSION}

One striking consequence of the self-limiting effect, of course, will be a remarkably narrow distribution in island size as experimentally observed. Large 3D islands will virtually stop further growth when their kinetic energy barriers become much higher than the thermal energy (kT). However, there is no significant resistance for the growth of small islands, consistent with the broad size distribution of islands at the very beginning of island growth, as shown in Fig. 1a. The self-limiting size at which the island growth starts to "feel" the self-limiting energy barrier depends sensitively on the misfit strain $\varepsilon$, as shown in the plot of Fig. 7b. This is consistent with the experimental results that highly strained $3 \mathrm{D}$ islands (such as InAs on GaAs) usually have smaller sizes than that of less strained islands (such as $\mathrm{Ge}_{0.5} \mathrm{Si}_{0.5}$ on $\mathrm{Si}$ ) when their growth reaches the self-limiting stage.

In addition to the self-limiting mechanism discussed above, the long-range strain field of a $3 \mathrm{D}$ island might also effect the adatom diffusion coefficient $\mathrm{D}_{s}$, and limit the flux reaching the island. Here we exclusively study such effect. For an isolated adatom on a planar surface, it is seen to exert forces on the other atoms from the "broken bond mechanism" as discussed by Duport et al. 12 At equilibrium, the total force is zero, but a dipole moment $m_{t}$ survives as the lowest-order multipole. The displacement field of the dipole, at distance $r$ away from the adatom, is given by 13

$$
\mathbf{u}_{r}=\frac{\left(1-v^{2}\right)}{\pi E} \frac{m_{r}}{r^{2}} \hat{\mathbf{r}}
$$

Here $E$ is the Young's modulus; $\hat{\mathbf{r}}$ is a unit vector pointing radially outward. For simplicity, we consider a circle-based pyramidal island with base radius $s$ and inclination angle $\theta$. the height gradient $\partial_{j} h(\mathbf{x})$ of the island surface exerts a force density $f_{j}=\sigma \partial_{j} h(\mathbf{x})$ at surface point $\mathbf{x}$, which can be written as

or

$$
f(r)=\sigma \tan (\theta) \hat{\mathbf{r}} \quad(r \leq s)
$$

$$
=0 \quad(r>s) \text {. }
$$


The dipole stress field of an adatom would interact with the large stress field of the 3D coherent island. The interaction energy for an adatom at a distance $R$ away from the center of the island can be calculated from the expression

$$
\begin{aligned}
U(R) & =-\int d \mathbf{r f}(\mathbf{r}) * \mathbf{u}_{\mathbf{r}}(\mathbf{r}-\mathbf{R}) \\
& =\frac{2\left(1-v^{2}\right) m_{r} \sigma \tan \theta}{\pi E} \int_{0}^{s} \int_{0}^{\pi} \frac{r(R \cos \phi-r) d r d \phi}{\left(R^{2}+r^{2}-2 r R \cos \phi\right)^{3 / 2}},
\end{aligned}
$$

where the 3D island is centered on the origin. A typical $U(R)$ vs. $R$ curve is shown in Fig. 9. It can be seen that the magnitude of the interaction energy is very small, <kT. By solving the rate equation for island growth, we find that the effect on the otherwise random diffusion of adatoms is insignificant. Therefore, the self-limiting effect should be dominated by the nucleation mechanism revealed in the previous section.

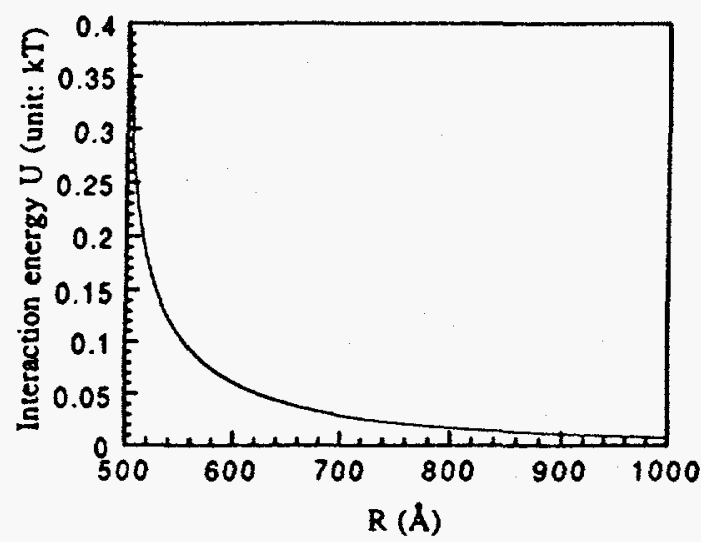

Fig. 9 The interaction energy $U$ (in the unit of $\mathrm{kT}=0.0735 \mathrm{eV}$ ) between a $3 \mathrm{D}$ island and an adatom vs. distance $R$. In the calculation, we assume $\varepsilon=0.02 ; \mathrm{m}_{\mathrm{r}}=0.07 \mathrm{~W}_{\text {coh, }}$ with cohesive energy $W_{\text {coh }}=3.0 \mathrm{eV} ; \mathrm{s}=500 \mathrm{~A}$; $\theta=11.2^{\circ}$.

Now, we discuss how this self-limiting effect operates during deposition. High supersaturation or direct vapor condensation on the island surface (flux $J_{v}$ in fig.4) will usually cause the island to grow under a far from equilibrium condition, and thus will degrade the size uniformity. However, when the island growth is dominated by the diffusion flux $J_{s}\left(\gg J_{v}\right)$, the self-limiting behavior could still operate to produce a narrow size distribution of $3 D$ islands. The kinetic picture can be described as follows: Due to the kinetic energy barrier to 3D island formation and the self-limiting effect of large existing 3D islands, highly supersaturated adatoms will form many small $2 \mathrm{D}$ islands between existing $3 \mathrm{D}$ islands to accommodate the continuously arriving atoms, as shown in the diagram of fig.10. Such 2D islands have been observed as mottled structure in biased secondary electron image, ${ }^{14}$ and more recently by in-situ STM. ${ }^{15}$ The 2D islands are unstable to dissolution upon fresh 3D island nucleation and/or dislocation introduction in the existing large 3D islands. In this sense, the mottled 2D structure acts as kinetic pathway or an atom reservoir for 3D island growth as it does in the annealing experiments. It has two important roles in mediating the growth kinetics of 3D islands. One is to effectively reduce the high supersaturation of adatoms, thus help to reduce the incoming adatom flux to the existing large 3D islands assisting the self-limiting effect. The other is to provide additional atoms to the flux, in addition to newly deposited atoms, for the growth of newly nucleated or small 3D islands, ${ }^{3}$ whose self-limiting effect are negligible. Both roles would contribute to achieving a narrow distribution in island sizes. 


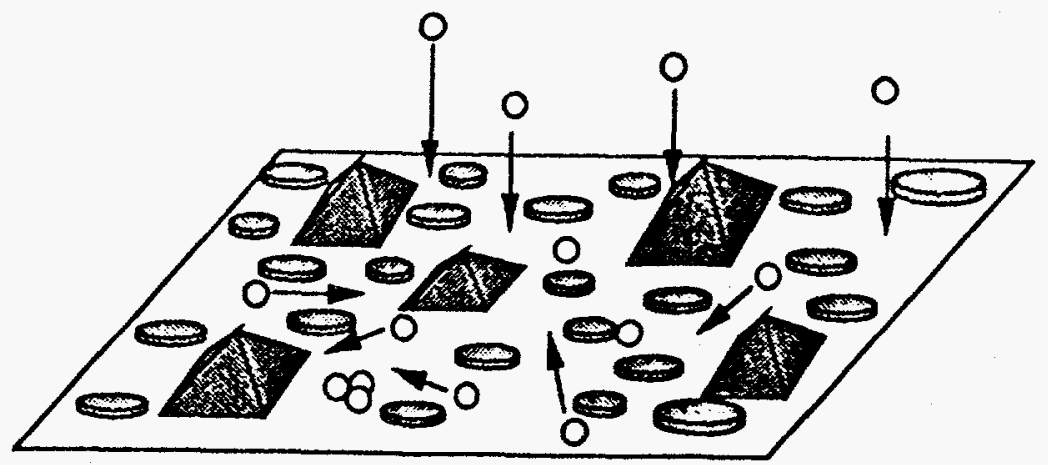

Fig. 10. A diagram showing the 3D island growth during deposition. Note the coexistence of $2 \mathrm{D}$ structures and $3 \mathrm{D}$ islands.

Finally, we discuss some possible events which will diminish the self-limiting effect on size uniformity of $3 \mathrm{D}$ islands. At the ripening or coalescence stage, there are no metastable 2D structures surrounding each 3D island. The direct thermodynamic interaction between $3 \mathrm{D}$ islands will cause large islands to grow at expense of small ones, thus resulting in a broad size distribution. The self-limiting effect can also be revoked by the introduction of a dislocation at the island edge, as it will suddenly reduce the local high stress field and the energy barrier. Subsequently the island grows very quickly until the high stress concentration built up again to self-limit its growth. The island grows slowly at this stage until the local stress concentration triggers another new dislocation. Then similar transient island growth occurs, followed by slow growth again, and so on. A cyclic growth of strained islands thus takes place following the successive formation of strain-relieving dislocations, exactly as observed by LeGoues et al. ${ }^{16}$

\section{CONCLUSIONS}

In summary, our studies have revealed an important self-limiting mechanism in strained 3D island growth. We believe that self-limiting behavior coupled with facetting can play a critical role in narrowing the size distribution of coherent islands. Our preliminary results demonstrate that the post-deposition annealing with careful control of $2 \mathrm{D} / 3 \mathrm{D}$ transition is a very promising approach to the fabrication of $3 D$ islands of uniform size and high areal number density. We expect that this new understanding of the growth kinetics will guide us to achieve optimal growth conditions for the fabrication of monosized quantum dot structures.

\section{ACKNOWLEDGMENTS}

This research was sponsored by the Division of Material Science, U. S. department of Energy under contract number DE-AC05-84OR21400 with Martin Marietta Energy System, Inc., and in part by an appointment to the Oak Ridge National Laboratory Postdoctoral Research Program administrated by Oak Ridge Institute for Science and Education. 


\section{REFERENCES}

1.M. Grundmann, et al. Phys. Rev. Lett. 74, 4043 (1995), and references therein.

2. D. J. Eaglesham, and M. Cerullo, Phys. Rev. Lett., 64, 1943 (1990).

3. D. Leonard, M. Krishnamurthy, C. M. Reaves, S. P. Denbaars, and P. M. Petroff, Appl. Phys. Lett. 63, 3203 (1993); D. Leonard, K. Pond, and P. M. Petroff, Phys. Rev. B50, 11687 (1994); D. Leonard, M. Krishnamurthy, S. Fafard, J. L. Marz, and P. M. Petroff, J. Vac. Sci. Technol. B12, 1063 (1994).

4. G. S. Solomon, J. A. Trezza, and J. S. Harris, Jr., Appl. Phys. Lett. 66, 991 (1995); 66, 3161 (1995).

5. J. M. Moison, F. Houzay, F. Barthe, L. Leprince, E. Andre and O. Vatel, Appl. Phys. Lett. 64, 196 (1994).

6. C. Priester and M. Lannoo, Phys. Rev. Lett. 75, 93 (1995).

7. V. A. Shchukin, N. Ledentsov, P. S. Kop'ev, and D. Bimberg. Phys. Rev. Lett. 75, 2968 (1995).

8. J. Tersoff, and R. M. Tromp, Phys. Rev. Lett. 70, 2782 (1993); J. Tersoff, and F. K. LeGoues, ibid, 72, 3570 (1994); J. Tersoff, ibid, 74, 4962(1995).

9. K. M. Chen, D. E. Jesson, S. J. Pennycook, T. Thundat, R. J. Warmack, to be published.

10. B. K. Chakraverty, J. Phys. Chem. Solids 28, 2401 (1967); and references therein.

11. D. E. Jesson, K. M. Chen, S. J. Pennycook, T. Thundat, R. J. Warmack, Science 268, 1161 (1995).

12. C. Duport, P. Nozieres, and J. Villain, Phys. Rev. Lett. 74, 134 (1995).

13. J. M. Rickman, and D. J. Srolovitz, Surf. Sci. 284, 211 (1993).

14. M. Krishnamurthy, J. S. Drucker, and J. A. Venables, J. Appl. Phys. 69, 6461 (1991).

15. B. R. Bennett, et al. in the Proceeding of 15th Annual Nonth American Conference on Molecular Beam Epitaxy, Sept. 17-20, 1995, in press.

16. F. K. LeGoues, M. C. Reuter, J. Tersoff, M. Hammer, and R. M. Tromp, Phys. Rev. Lett. 73, 300 (1994). The shape change of a 3D island could also contribute to the rapid lateral growth of the island following the dislocation introduction, as suggested by the authors. However, the slow growth preceding the dislocation nucleation is an indication of the self-limiting behavior discussed in this paper.

\section{DISCLAIMER}

This report was prepared as an account of work sponsored by an agency of the United States Government. Neither the United States Government nor any agency thereof, nor any of their employees, makes any warranty, express or implied, or assumes any legal liability or responsibility for the accuracy, completeness, or usefulness of any information, apparatus, product, or process disclosed, or represents that its use would not infringe privately owned rights. Reference herein to any specific commercial product, process, or service by trade name, trademark, manufacturer, or otherwise does not necessarily constitute or imply its endorsement, recommendation, or favoring by the United States Government or any agency thereof. The views and opinions of authors expressed herein do not necessarily state or reflect those of the United States Government or any agency thereof. 